\title{
Plasma Amino Acids in Attention Deficit Disorder
}

\author{
R.A. Bornstein, Glen B. Baker, Alan Carroll, Gordon King, James T.F. Wong, \\ and Alan B. Douglass
}

Received April 2, 1990; revised version received June 29, 1990; accepted July 21, 1990.

\begin{abstract}
This study examines plasma amino acids in a group of 28 patients meeting $D S M-I I I$ criteria for attention deficit disorder (ADD) and 20 control subjects. Compared with controls, the ADD subjects had significantly lower levels of phenylalanine, tyrosine, tryptophan, histidine, and isoleucine. These data suggest a general deficit in amino acid transport, absorption, or both.
\end{abstract}

Key Words. Attention deficit disorder, phenylalanine, amino acids.

Abnormalities in a number of neurotransmitter systems have been investigated in regard to the pathophysiological mechanisms underlying attention deficit disorder (ADD). Studies in various body fluids have failed to identify consistent differences between ADD subjects and controls (Zametkin and Rapoport, 1987) with regard to dopamine, norepinephrine, serotonin, or the metabolites of these neurotransmitters. Zametkin et al. ( $1984 a, 1984 b)$ reported decreased urinary $\beta$-phenylethylamine (PEA) in ADD subjects relative to normals, but no difference in urinary phenylacetic acid (the acid metabolite of PEA). The same study found no differences between controls and children with ADD in urinary levels of $p$-tyrosine or phenylalanine. To our knowledge, there have been no comprehensive studies of amino acids in plasma as opposed to urine. The current investigation was therefore performed to examine this aspect of the disorder.

\section{Methods}

The study group consisted of 28 subjects ( 23 males, 5 females) who met $D S M-I I I$ criteria for ADD (American Psychiatric Association, 1980). Diagnoses were established by board-certified child psychiatrists. These subjects were compared with a group of 20 controls $(8$ males, 12 females) who were recruited from the community. Most were the children of hospital staff. The control subjects underwent a thorough history for neurological or psychiatric symptoms. Only patients with no history of evaluation or treatment for any psychiatric and neurological disease were included. All control subjects were screened for histories of neurological or psychiatric disorders including ADD. All subjects were Caucasian. The mean age of the ADD subjects was

R.A. Bornstein, Ph.D., is Associate Professor of Psychiatry, Neurology, and Neurosurgery, The Ohio State University, Columbus, OH. Glen B. Baker, Ph.D., is Professor and Co-Director, Neurochemical Research Unit, University of Alberta, Alberta, Canada. At the time this work was done, Alan Carroll, M.D., Gordon King, M.D., James T.F. Wong, Ph.D., and Alan B. Douglass, M.D., were in the Department of Psychiatry, University of Alberta, Alberta, Canada. Dr. Wong is now a postdoctoral fellow in the Department of Psychiatry at the University of British Columbia, and Dr. Douglass is in the Department of Psychiatry, University of Michigan. (Reprint requests to Dr. R.A. Bornstein, Neuropsychology Laboratory, The Ohio State University, 473 W. 12th Ave., Columbus, OH 43210, USA.)

0165-1781/90/\$03.50 @ 1990 Elsevier Scientific Publishers Ireland Ltd. 
9.6 years $(\mathrm{SD}=2.4)$, and that of the control subjects was 10.8 years $(\mathrm{SD}=3.8)$; these values did not differ significantly $(t=1.27, p>0.20)$. The proportion of males and females in the two groups was significantly different $\left(\chi^{2}=7.31, p<0.01\right)$.

All subjects were placed on a low amine diet for the 48 hours preceding sample collection. Venous blood samples for the isolation of plasma were obtained by a nurse practitioner after an overnight fast. Samples were collected at the same time (8-9 a.m.). In addition to blood samples, a 24-hour urine sample was obtained. All patients were free of medication at the time of sample collection. Urine collections were obtained at home under parental supervision. The groups did not differ in urine volume $(t=0.41, p>0.50)$ or creatinine $(t=0.41, p>0.50)$. Electron capture gas chromatography was used for analysis of plasma amino acid levels (Yeung et al., 1986) after extraction and derivitization. Analyses were performed for tryptophan (TRP), $p$-tyrosine (TYR), phenylalanine (PHE), histidine (HIS), valine (VAL), and isoleucine (ILEU). These are classified as large neutral amino acids (LNAAs) and compete for transport across the bloodbrain barrier (Pardridge and Oldendorf, 1975; Wurtman, 1978).

It is also of interest to explore the relationship between these amino acids and symptoms of ADD. It was hypothesized that if amino acid abnormalities contribute to the pathophysiology of ADD, it might be possible to demonstrate correlations between amino acid levels and ADD symptoms. To address this question, parents of the subjects completed the Conners Parent Questionnaire (Conners, 1970).

\section{Results}

The initial data analysis consisted of comparison of group means on each of the amino acids using $t$ tests for independent samples. Table 1 demonstrates that the ADD patients have significantly lower levels of a number of amino acids, including TYR, HIS, and ILEU. On several measures, the group variances were significantly different, which necessitated the use of nonparametric procedures. For those variables, MannWhitney $U$ tests were performed. The results of these analyses are also presented in Table 1, which shows significantly lower levels of TRP and PHE in the ADD subjects. These data suggest consistently decreased plasma levels of amino acids in the ADD

Table 1. Plasma and urinary amino acids 1 in attention deficit disorder (ADD) and control groups

\begin{tabular}{|c|c|c|c|c|c|c|}
\hline & \multicolumn{2}{|c|}{ ADD } & \multicolumn{2}{|c|}{ Control } & \multirow[b]{2}{*}{$t 2$} & \multirow[b]{2}{*}{$M-W^{2}$} \\
\hline & Mean & SD & Mean & SD & & \\
\hline \multicolumn{7}{|l|}{ Plasma } \\
\hline Histidine & 90.4 & 63.1 & 143.0 & 88.2 & 0.025 & \\
\hline Isoleucine & 57.2 & 73.1 & 119.9 & 52.1 & 0.005 & \\
\hline Tryptophan & 58.1 & 97.6 & 104.2 & 71.9 & 0.09 & 0.002 \\
\hline Tyrosine & 111.9 & 109.0 & 280.3 & 175.8 & 0.001 & 0.001 \\
\hline Phenylalanine & 93.0 & 84.2 & 126.9 & 53.7 & 0.13 & 0.005 \\
\hline Alanine & 536.9 & 405.0 & 766.0 & 381.6 & 0.06 & \\
\hline Valine & 188.7 & 87.4 & 177.2 & 160.5 & 0.78 & 0.19 \\
\hline \multicolumn{7}{|l|}{ Urine } \\
\hline Tyrosine & 203.8 & 112.3 & 240.1 & 147.2 & 0.38 & \\
\hline Phenylalanine & 74.4 & 55.5 & 72.0 & 36.0 & 0.88 & \\
\hline
\end{tabular}

Note. $M-W=$ Mann-Whitney.

1. Micromoles/liter in plasma, micromoles/g creatinine in urine.

2. $\rho$ values. 
sample. Consistent with previous studies (Zametkin et al., 1984a, 1984b; Baker et al., in press), there was no difference between the groups in urinary levels of PHE or TYR.

It was considered possible that the difference in the proportion of males and females in the two groups could have influenced these results. Therefore, a two-way analysis of variance (group $\times$ sex) was performed for all measures on which a significant difference had been found. The results of these analyses revealed no significant main effects for sex, and no group $\times$ sex interactions. Therefore, differences in the sexual composition of the groups do not appear to account for the differences in amino acid levels. Inclusion of age as a covariate in the analyses did not alter the pattern of significant results.

Table 2 presents mean scores from the Conners Parent Questionnaire (93-item) for the ADD groups. Pearson correlation coefficients were computed to explore the relationship between ADD symptoms and amino acid levels. Table 2 also presents the correlations between the Impulsive/ Hyperactive Scale of the Conners Parent Questionnaire and the amino acid levels. It can be seen that there are consistent negative correlations, indicating that lower levels are associated with more prominent symptoms. Review of the correlations indicates that six of the seven correlations with plasma amino acid levels are negative (binomial probability $p<0.05$ ).

Table 2. Mean Conners Parent Questionnaire scores 1 and Pearson correlations with plasma amino acid levels

\begin{tabular}{lccccccccc}
\hline & & & \multicolumn{8}{c}{ Mean } & SD & HIS & ILEU & TRP & TYR & PHE & ALA & VAL \\
\hline Conduct & 47.4 & 26.8 & -0.22 & -0.31 & 0.07 & -0.35 & -0.31 & -0.25 & -0.12 \\
Anxiety & 25.7 & 16.2 & -0.00 & -0.04 & -0.21 & -0.00 & -0.03 & 0.08 & -0.28 \\
Impulsivity & 54.0 & 26.4 & -0.17 & -0.35 & 0.01 & -0.36 & -0.38 & -0.24 & -0.38 \\
Learning & 35.3 & 23.0 & 0.422 & 0.23 & 0.34 & 0.19 & 0.16 & 0.19 & 0.34 \\
$\begin{array}{l}\text { Psycho- } \\
\text { somatic }\end{array}$ & 17.1 & 16.3 & 0.13 & 0.31 & -0.26 & 0.30 & 0.33 & 0.422 & 0.492 \\
$\begin{array}{l}\text { Perfectionism } \\
\text { Antisocial }\end{array}$ & 11.1 & 27.2 & 0.17 & -0.20 & 0.00 & -0.11 & -0.14 & -0.13 & -0.03 \\
$\begin{array}{l}\text { Muscular } \\
\text { tension }\end{array}$ & 19.4 & 26.5 & 0.30 & 0.03 & 0.02 & 0.05 & 0.07 & 0.07 & 0.20 \\
\hline
\end{tabular}

Note. HIS $=$ histidine. ILEU $=$ isoleucine. TRP $=$ tryptophan. TYR = tyramine. PHE = phenylalanine. $A L A=$ alanine. $\mathrm{VAL}=$ valine.

1. The Conners scores represent the percentage of possible points for each scale (total endorsed/total possible).

2. $\rho<0.05$.

\section{Discussion}

The present study demonstrates consistently low levels of LNAAs in plasma in children with ADD. In addition, the lack of urinary differences in PHE and TYR replicates previous reports (Zametkin et al., 1984a, 1984b; Baker et al., in press). The amino acids examined in this study all compete for the same transport system across 
the blood-brain barrier. The finding of lower plasma levels for virtually all the LNAAs (except VAL) suggests that there may be a generalized abnormality in absorption or in the transport system itself that alters the plasma levels of the competing amino acids. This would be in contrast to other disorders such as phenylketonuria in which the high level of transport of one amino acid (PHE) interferes with the transport of the other amino acids (Proud et al., 1989). The significance of the lowered plasma levels of the LNAAs is difficult to ascertain without knowing if the brain levels are increased, decreased, or unchanged. The rather marked lowering of the plasma LNAAs indicate that cerebrospinal fluid measurements of these substances would be a fruitful line of research. Recent reports suggesting that brain uptake of LNAAs is under control of $\beta$-adrenergic receptors also appear to be relevant (Edwards and Sorisio, 1988; Ericksson and Carlsson, 1988; Edwards et al., 1989).

The suggestions from these data of a general amino acid absorption or transport abnormality in ADD may provide some insight into previous biochemical studies of these patients. The amino acids TYR, TRP, and PHE represent the precursors for catecholamines, indoleamines (5-hydroxytryptamine and tryptamine), and $\beta$-phenylethyl]amine, respectively. It is possible that the reports of decreased levels of 3 methoxy-4-hydroxyphenylglycol (a major metabolite of norepinephrine) in ADD children (Shekim et al., 1979; Yu-cun and Yu-feng, 1984) may be related to a more primary deficit in the amino acid precursors (TYR and PHE). This explanation is not completely satisfactory since urinary studies of dopamine metabolites (homovanillic acid and 3-methoxytyramine) with the same amino acid precursors have not shown differences between control and ADD subjects (Baker and Bornstein, unpublished). Several studies (Coleman, 1971; Bhagauan et al., 1975) have also shown decreased levels of 5-hydroxytryptamine in serum or whole blood in ADD subjects.

The possibility of a generalized amino acid absorption or transport abnormality may also provide some insights to the various pharmacological treatment approaches in ADD. In studies reviewed elsewhere (Klein, 1987), the pharmacological interventions that have been most effective in treatment of ADD are those which augment catecholaminergic transmission. These include mixed dopamine and norepinephrine agonists as well as the monoamine oxidase inhibitor tranylcypromine (Zametkin et al., 1985). In contrast, approaches using administration of the precursor of serotonin (L-tryptophan) have not been particularly effective in the treatment of ADD (Nemzer et al., 1986).

As pointed out by Zametkin and Rapoport (1987), it is increasingly unlikely that any single neurotransmitter system or model can account for the diverse pharmacological responses that have been reported in the treatment of ADD. Furthermore, many of the studies used to investigate treatment are based on very small sample sizes. Given the heterogeneity of treatment response in ADD, it is possible that there are subgroups of patients with different underlying pathophysiologies. One potentially useful approach in treatment studies of new or previously established interventions might be to examine the characteristics of patients who do or do not respond to a specific treatment modality. The findings of the present report suggest that future studies should include an examination of plasma and cerebrospinal fluid levels of LNAAs. 
Acknowledgments. Funds were provided by the Alberta Provincial Mental Health Advisory Council (PMHAC) and the National Institute of Mental Health (MH-42209, R.A.B.). The authors are grateful to J. van Muyden, T. Bazylewich, and S. Ashton for providing expert assistance and to W.G. Dewhurst, A.J. Greenshaw, and P.R. Paetsch for useful discussions. Versions of this manuscript were prepared by $\mathbf{S}$. Omura and $\mathbf{S}$. Craley.

\section{References}

American Psychiatric Association. DSM-III: Diagnostic and Statistical Manual of Mental Disorders. 3rd ed. Washington, DC: APA, 1980.

Baker, G.B.; Bornstein, R.A.; Rouget, A.C.; Ashton, S.E.; van Muyden, J.C.; and Coutts, R.T. Phenylethylaminergic mechanisms in attention deficit disorder. Biological Psychiatry, in press.

Bhagauan, H.N.; Coleman, M.; and Coursina, D.B. The effect of pyridoxine hydrochloride on blood scrotonin and pyridoxal phosphate contents in hypcractive children. Pediatrics, 55:437-441, 1975.

Coleman, M. Serotonin concentrations in whole blood of hyperactive children. Journal of Pediatrics, 78:986-990, 1971.

Conners, C.K. Symptom patterns in hyperkinetic, neurotic, and normal children. Child Development, 41:667-682, 1970.

Edwards, D.J., and Sorisio, D.A. Effects of imipramine on tyrosine and tryptophan are mediated by $\beta$-adrenoceptor stimulation. Life Sciences, 42:853-862, 1988.

Edwards, D.J.; Sorisio, D.A.; and Knopf, S. Effects of the $\beta_{2}$-adrenoceptor agonist clenbuterol on tyrosine and tryptophan in plasma and brain of the rat. Biochemical Pharmacology, 38:2957-2965, 1989.

Eriksson, T., and Carlsson, A. $\beta$-Adrenergic control of brain uptake of large neutral amino acids. Life Sciences, 42:1583-1589, 1988.

Klein, R.G. Pharmacotherapy of childhood hyperactivity: An update. In: Meltzer, H.Y., ed. Psychopharmacology: The Third Generation of Progress. New York: Raven Press, 1987. pp. 1215-1224.

Nemzer, E.D.; Arnold, L.E.; Votolato, N.A.; and McConnell, H. Amino acid supplementation as therapy for attention deficit disorder. Journal of the American Academy of Child and Adolescent Psychiatry, 25:509-513, 1986.

Pardridge, W.M., and Oldendorf, W.H. Kinetic analysis of blood-brain barrier transport of amino acids. Biochemical and Biophysical Research Communications, 401:128-136, 1975.

Proud, V.K.; Hsia, Y.E.; and Wolf, B. Disorders of amino acid metabolism. In: Siegel G.; Agranoff, B.; Albers, R.W.; and Molinoff, P.B., eds. Basic Neurochemistry. 4th ed. New York: Raven Press, 1989. pp. 733-763.

Shekim, W.O.; DeKirmenjian, H.; and Chapel, J.L. Urinary MHPG excretion in minimal brain dysfunction and its modification by $d$-amphetamine. American Journal of Psychiatry, 136:667-671, 1979.

Shekim, W.O.; Javaid, J.; Davis, J.M.; and Bylund, D.B. Urinary MHPG and HVA excretion in boys with attention deficit disorder and hyperactivity treated with $d$-amphetamine. Biological Psychiatry, 18:707-714, 1983.

Wurtman, R.J. Effects of nutrients and circulating precursors on the synthesis of brain neurotransmitters. In: Garattini, S., and Samanin, R., eds. Central Mechanisms of Anorectic Drugs. New York: Raven Press, 1978. pp. 267-294.

Yeung, J.M.; Baker, G. B.; and Coutts, R.T. Simple automated gas chromatographic analysis of amino acids and its application to brain tissue. Journal of Chromatography, Biomedical Applications, 378:293-304, 1986.

Yu-cun, A., and Yu-feng, W. Urinary 3-methoxy-4-hyd roxyphenylglycol sulfate excretion in seventy-three school children and adolescents with psychiatric disorders. Biological Psychiatry, 19:861-870, 1984. 
Zametkin, A.J.; Brown, G.L.; Karoum, F.; Rapoport, J.L.; Langer, D.H.; Chuang, L.W.; and Wyatt, R.J. Urinary phenethylamine response to $d$-amphetamine in 12 boys with attention deficit disorder. American Journal of Psychiatry, 141, 1055-1058, 1984.

Zametkin, A.J.; Karoum, F.; Rapoport, J.L.; Brown, G.L.; and Wyatt, R.J. Phenylethylamine excretion in attention deficit disorder. Journal of the American Academy of Child and Adolescent Psychiatry, 23:310-314, $1984 b$.

Zametkin, A.J., and Rapoport, J.L. Neurobiology of attention deficit disorder with hyperactivity: Where have we come in 50 years? Journal of the American Academy of Child and Adolescent Psychiatry, 26(5):676-686, 1987.

Zametkin, A.J.; Rapoport, J.L.; Murphy, D.L.; Linnoila, M.; and Ismond, D. Treatment of hyperactive children with monoamine oxidase inhibitors: I. Clinical efficacy. Archives of General Psychiatry, 42:962-969, 1985. 